\title{
Convention on the means of prohibiting and preventing the illicit import, export and transfer of ownership of cultural property
}

UNESCO

Follow this and additional works at: http://digitalcommons.law.scu.edu/cultprop

Part of the International Law Commons

\section{Automated Citation}

UNESCO, "Convention on the means of prohibiting and preventing the illicit import, export and transfer of ownership of cultural property" (1970). Protection of Cultural Property in the Event of Armed Conflict. Paper 18.

http://digitalcommons.law.scu.edu/cultprop/18 
United Nations Educational. Scientific and Cultural Organzation Organización de las Naciones Unidas para la Educación, la Ciencia y la Cultura

Organisation des Nations Unies pour l'éducation, la science et la culture Организация объединенных наций по вопросам образования, науки и кц.льтуры

Convention on the means of prohibiting and preventing the illicit import, export and transfer of ownership of cultural property

adopted by the General Conference at its sixteenth session Paris, 14 November 1970

Convención sobre las medidas que deben adoptarse para prohibir e impedir la importación, la exportación y la transferencia de propiedad ilicitas de bienes culturales aprobada por la Conferencia General en su decimosexta reunion Paris, 14 de noviembre de 1970

Convention concernant les mesures à prendre pour interdire et empêcher l'importation, l'exportation et le transfert de propriété illicites des biens culturels adoptée par la Conférence générale à sa seizième session Paris, le 14 novembre 1970

\section{Конвенция о мерах, направленных}

на запрещение и предупреждение незаконного ввоза, вывоза и передачи права собственности на культурные ценности принятая Генеральной хонференцией на пестнадцатой сессии Париж, 14 ноября 1970 r. 
CONVENTION ON THE MEANS OF PROHIBITING AND PREVENTING THE UTICIT IMPORT, EXPORT AND TRANSFER OF OWNERSHIP OF CULTURAL PROPERTY
CONVENCION SOBRE LAS

MEDIDAS QUE DEBEN ADOPTARSE PARA

PROHIBIR E IMPEDIR LA IMPORTACION:

LA EXPORTACION Y LA TRANSFERENCIA

DE PROPIEDAD ILICITAS DE BIENES CULTURALES
The General Conference of the United Nations Educational, Scientific and Cultural Organization, meeting in Paris from 12 October to 14 November 1970, at its sixteenth session.

Recalling the importance of the provisions contained in the Declaration of the Principles of International Cultural Co-operation, adopted by the General Conference at its fourteenth session,

Considering that the interchange of cultural property among nations for scientific, cultural and educational purposes increases the knowledge of the civilization of Man, enriches the cultural life of all peoples and inspires mutual respect and appreciation among nations,

Considering that cultural property constitutes one of the basic elements of civilization and national culture, and that its true value can be appreciated only in relation to the fullest possible information regarding its origin, history and traditional setting,

Considering that it is incumbent upon every State to protect the cultural property existing within its territory against the dangers of theft, clandestine excavation, and illicit export,

Considering that, to avert these dangers, it is essential for every State to become increasingly alive to the moral obligations to respect its own cultural heritage and that of all nations,

Considering that, as cultural institutions, museums libraries and archives should ensure that their collections are built up in accordance with universally recognized moral principles,

Considering that the illicit import, export and transfer of ownership of cultural property is an obstacle to:that understanding between nations which it is part of Unesco's mission to promote by recommending to interested States, international conventions to this end,

Considering that the protection of cultural heritage can be effective only if organized both nationally and internationally among States working in close co-operation,

Considering that the Unesco General Conference adopted a Recommendation to this effect in 1964 ,

Having before it further proposals on the means of prohibiting and preventing the illicit import, export and transfer of ownership of cultural property, a question which is on the agenda for the session as item 19 ,
La Conferencia General de la Organizacion de las Naciones Unidas para la Educacion, la Ciencia y la Cultura, en su $16 a$. reunion, celebrada en Parfs, del 12 de octubre ai 14 de noviembre de 1970 ,

Recordando la importancia de las disposiciones de la Declaración de los principios de la cooperación cultural internacional que la Conferencia General aprobo en su $14 a$. reunion,

Considerando que el intercambio de bienes culturales entre las naciones con fines cientrficos, culturales y educativos aumenta los conocimientos sobre la civilizacion humana, enriquece la vida cultural de todos los pueblos e inspira el respeto mutuo y la estima entre las naciones,

Considerando que los bienes culturales son uno de los elementos fundamentales de la civilizacion y de la cultura de los pueblos, y que solo adquieren su verdadero valor cuando se conocen con la mayor precision su origen, su historia y su medio,

Considerando que todo Estado tiene el deber de proteger el patrimonio constituido por los bienes culturales existentes en su territorio contra los peligros de robo, excavacion clandestina y exportacion ilfcita,

Considerando que para evitar esos peligros es indispensable que todo Estado tenga cada vez más conciencia de las obligaciones morales inherentes al respeto de su patrimonio cultural y del de todas las naciones,

Considerando que los museos, las bibliotecas y los archivos, como instituciones culturales, deben velar por que la constitucion de sus colecciones se base en principios morales universalmente reconocidos,

Considerando que la importacion, la exportacion y la transferencia de propiedad ilfcitas de los bienes culturales dificultan la comprension mutua de las naciones que la Unesco tiene el deber de favorecer, entre otras formas, recomendando a los Estados interesa dos que concierten convenciones internacionales con ese objeto,

Considerando que, para ser eficaz, la proteccion del patrimonio cultural debe organizarse tanto en el plano nacional como en el 

ILLICITES DES BIENS CULTURELS
КОНВЕНЦИЯ О МЕРАX,

НАПРАВЛЕННЫХ НА ЗАПРЕШЕНИЕ И ПРЕДУПРЕЖДЕНИЕ НЕЗАКОННОГО ВВОЗА, ВЬВОЗА И ПЕРЕДАЧИ ПРАВА СОБСТ ВЕННОСТИ НА КУЛЬТУРНЫЕ ЦЕННОСТИ
La Conférence générale de l'Urganisation des Nations Unies pour l'éducation, la science et la culture, reunie à Paris, du 12 octobre au 14 novembre 1970 en sa seizieme session,

Rappelan: ''ımportance des dispositions de la Déclara:ion des principes de la coopération culturelle internationale adoptée par la Conférence générale à sa quatorzième session,

Considérant que l'échange de biens culturels entre nations à des fins scientifiques, culturelles et éduca:ives approfondit la connaissance de la civilisat:on humaine, enrichit la vie culturelle de tous les peuples et fait naftre le respect et l'es time mutuels entre les nations,

Considérant que les biens culturels sont un des éléments fondamentaux de la civilisation et de la culture ces peuples, et qu'ils ne prennent leur valeur reelle que si leur origine, leur histoire et leur environzement sont connus avec la pius grande precision,

Consideran: que chaque Etat a le devoir de protéger le pa:rimoine constitué par les biens culturels existant sur son territoire contre les dangers de vol, de fouilles clandestines et d'expor:ation illic:ee,

Consideran: que, pour parer à ces dangers, il est indispensable que chaque Etat prenne davantage conscience des obligations morales touchant au respec: de son patrimoine culturel comme de celui de toutes les nations,

Considerant que les musées, les bibliothèques et les archives, en tant qu'institutions culturelles, doiven: ve:Hler à ce que la constitution de leurs collec:tons soit fondee sur des principes moraux universellement reconnus,

Considerant que l'importation, l'exportation et le transfer: de propriété illicites des biens culturels entravent la compréhension mutuelle des nations que l'Unesco a le devoir de favoriser, entre autres en recommandant aux Etats intéres sés des conventions internationales à cet effet,

Considérant que; pour etre efficace, la protection du patrimoine culturel doit etre organisée tant sur le plan national qu'international et exige une étroite collaboration entre les Etats,

Considerant que la Conférence générale de l'Unesco a déjà adopté, en 1964 , une recommandation à cet effet,

Etant saisie de nouvelles propositions concernant
Генеральная конференция Органнзашии объеднненных наций по вопросам образования, науки и культуры, собравщаяся в Парнже с 12 октября по 14 ноября 1970 г. на свор шестнадатур сессио, напоминая о важности положений Декларачин принцилов международного сотру дничества в области культуры, принятой на четырнаднатой сессии Генеральной конференции (1966 г.), считая, что обмен культурными ценностями мехду странами для целей образования, наукн и культуры растиряет знания о человеческой цивилизацин, обогащает культурнур жизнь всех народов и вызывает взаимное уважение и понимание между странахи,

счнтая, что культурные ценности являртся одним из основных элементов цивилизация н культуры народов и что они прнобретарт свор подлинуу ценность только в том случае, если точно известны их происхождение, исторня и окрухарщая среда,

считая, что каждое государство обязано охранять достояние, состоящее из находящихся на его террнтория культурных ценностей, от опасностей кражи, тайных расхопок и незаконного вывоза,

считая, что для предупреждения этих опасностей необходимо, чтобы каждое государство еше больще прониклось сознанием моральных обязательств в отношении как своего культурного достояния, так и культурного достояния всех народов,

считая, что музеи, библиотеки и архнвы, являясь учреждениями культуры, должны заботиться о том, чтобы их коллекции создавались на основе всеобщепрнзнанных моральных принципов,

счнтая, что незахонные ввоз, вывоз и передача права соб́ственности на культурные ценности наносят ущерб взаимопониманию между народами, содействовать которому ЮНЕСКО обязана, в частности, рекомендуя заинтересованным государствам соответствуюшие международные конвеншии,

счнтая, что для того, чтобы быть эффективной, охрана культурного достояния должна 6 ыть организована как в национальном, тах и в мехдународном масштабах и требует тесного сотрудничества между государствами,

принимая во внимание, что Генеральная конференция ЮНЕСКО в 1964 г. уже приняла рекомендачих по данному вопросу, 
Having deciued, at its fifteenth session, that this question should be made the subject of an international :onvention.

Adopts this -onvention on the fourteenth day of November 1970

\section{Article 1}

For the puryoses of this Convention, the term "cultural property" means property which, on religious or se zular grounds, is specifically designated by each jtate as being of importance for archaeology, prehistory, history, literature, art or science and whic : belongs to the following categories:

(a) Rare col.ections and specimens of fauna, flora, minerais and anatomy, and objects of palaeontclogical interest;

(b) property relating to history, including the history of science and technology and military and social history, to the life of nationalleaders, thinkers, scientists and artists and to events of national importance;

(c) products of archaeological excavations (including regular and clandestine) or of archaeological discoveries;

(d) elements of artistic or historical monuments or archaeological sites which have been dismembered;

(e) antiquities more than one hundred years old, such as inscriptions, coins and engraved seals;

(f) objects of ethnological interest;

(g) property of artistic interest, such as:

(i) pictures, paintings and drawings produced entirely by hand on any support and in any material (excluding industrial designs and manufactured articles decorated by hand); original works of statuary art and sculpture in any material;

(iii) original engravings, prints and lithographs;

(iv) original artistic assemblages and montages in any material:

(h) rare manuscripts and incunabula, old books, documents and publications of special interest (historical, artistic, scientific, literary, etc. ) singly or in collections;

(i) postage, revenue and similar stamps, singl or in collections;

(j) archives, including sound, photographic and cinematographic archives; internacional, $y$ que exige una estrecha cola boracion entre los Estados.

Considerando que la Conferencia General de la Unesco aprobo ya en 1964 una Recomenda. cion con este objeto,

Habiendo examinado nuevas propuestas relativas a las medidas destinadas a prohibir e impedir la importacion, la exportacion y la trans ferencia de propiedad illcitas de bienes culturales, cuestion que constituye el punto 19 del orden del dia de la reunión,

Después de haber decidido, en la $15 a$. reunion, que esta cuestion serra objeto de una convención internacional, aprueba el día catorce de noviembre de 1970, la presente Convencion.

\section{Articulo primero}

Para los efectos de la presente Convención se considerarán como bienes cuiturales los objetos que, por razones religiosas o profanas, hayan sido expreșamente designados por cada Estado como de importancia para la arqueologia, la prehistoria, la historia, la literatura, el arte - la ciencia y que pertenezcan a las categorfas enumeradas a continuacion:

a) las colecciones y ejemplares raros de zoologla, botánica, mineralogfa, anatomfa, y los objetos de interes paleontologico;

b) los bienes relacionados con la historia, con inclusion de la historia de las ciencias y de las técnicas, la historia militar y la historia social, as1 como con la vida de los dirigentes, pensadores, sabios y artistas nacionales y con los acontecimientos de importancia nacional;

c) el producto de las excavaciones (tanto autorizadas como clandestinas) o de los descubrimientos arqueologicos:

d) los elementos procedentes de la desmembracion de monumentos artisticos o historicos $y$ de lugares de interes arqueologico;

e) antiguedades que tengan más de 100 anos, tales como inscripciones, monedas y sellos grabados

f) el material etnologico;

g) los bienes de interés artístico tales como: i) cuadros, pinturas y dibujos hechos ente ramente a mano sobre cualquier soporte y en cualquier material (con exclusion de los dibujos industriales y de los articu-

ii) los manufacturados decorados a mano); rio y de esculturanales de arte estatuario y de escultura en cualquier material;

iii) grabados, estampas y litograffas originales;

iv) conjuntos y montajes artisticos originales en cualquier material.

h) manuscritos raros e incunables, libros, documentos y publicaciones antiguos de interés 
les mesures à prendre pour interdire et empecher l'importation, l'exportation et le transfer: de propriété illicites des biens culturels, ques tion qui constitue le point 19 de l'ordre du jour de la session.

Après avoir décidé, lors de sa quinzieme session, que cette question ferait l'objet d'une convention. internationale, adopte, ce quatorzieme jour de novembre 1970 , la présente Convention. получнв новые предлохения, касаюшнеся мер, направленных на запрещение н предупреждение незаконных ввоза, вывоза и передачи права собственности на хультурные ценности (пункт 19 повестхи дня сессин),

решнв на своей пятнашатой сессии, что данный вопрос явнтся предметом международной конвенции, принимает четырнаднатого ноября 1970 Г. настояшую Конвеншнх.

\section{Article premier}

Aux fins de la présente Convention sont considérés comme biens culturels les biens qui, à titre religieux ou profane, sont désignés par chaque Eiat comme étant d'importance pour l'archéologie, la préhistorre. 'histoire, la littérature, l'ar: ou la science, e: qui appartiennent aux catégories c:après :

(a) collections et spécimens rares de zooiogie, de botanique, de minéralogie et d'anatomie; objets présentant un intéret paléontologique :

(b) les biens concernant l'histoire, y compris l'histonte des sciences et des techniques, l'histoire miltaire et sociale ainsi que la vie des dir:gear:s, penseurs, savants et artistes nationaux, et les événements d'importance nationale.

(c) le procu: des fouilles archéologiques (régulières e: clandestines) et des découvertes arcneolog:ques :

(d) les éements provenant du démembrement de monuments aristiques ou historiques et des sites archéologiques ;

ie) objets d'antiquité ayant plus de cent ans d'áge, tels que inscriptions, monnaies et sceaux graves:

(f) le mater:e: etinologique

(g) ies biens c'intéret artistique tels que :

(i) tabiealux, peintures et dessins faits entièrement à la main sur tout support et en toutes matières (à l'exclusion des dessins indus trieis et des articles manufacturés décorés a la main ;

(ii) productions originales de l'art statuaire et de la sculpture, en toutes matières ;

(iii) gravures, estampes et lithographies originaies :

(iv) assemblages et montages artistiques originaux, en toutes matieres ;

(h) manuscrits rares et incunables, livres, documents et publications anciens d'intéret spécial (historique, artistique, scientifique, littéraire, etc. ) isoiés ou en collections :

(i) timbres-poste, timbres fiscaux et analogues, isolés ou en collections :

\section{Cтатья 1}

Пляя целей настояпей Конвеншни культурными ченностями считахтся пенности религнозного или светского характера, которые рассматривахтся кахдым государством ках представляошие значение лля археологин, донсторнчесхого пернода, историн, литературы, нсхусства н науки и которые относятся к перечнсляемым нихе категорням:

(a) редкие холлекцин и образшы флоры и фаунь, минералогин, анатомин н предметы, представляюшие ннтерес для палеонтология;

(b) ценности, хасахшиеся нсторин, вхлочая исторно наукн и техники, историю войн и обществ, а таххе сзязанные с жизньх нашнональных деятелей, мыслителей, ученых и артнстов н с крулными национальными соб̆ытнямн;

(c) археологичесхие находки (включая об̆ычные и тайные) и археологические открытия;

(d) состазные части расчлененных художественных н исторических памятников и археологических мест;

(e) старннные предметы более чем 100-летней давности, такие ках надписи, чеханные монетsl и печати;

(:! этнологические матерналы;

(g'. художественные ценности, такне как:

(1) полотна, . картины и рнсунки целиком ручной работы на любой основе и из любых матерналов (за исключением чертежей и промыщленных нзделий, украшенных от руки);

(ii) орнгинальные пронзведения схульптурного нсхусства нз любых материалов;

(tii) оригинальные гравюры, эстампы и литографик;

(iv) оригинальные художественные подбоорки н монтажи из любых матерналов;

(h) редкне рукописи и инкунабулы, старннные книги, документы и нздания, представляхшие особый интерес (исторнчесхий, художественный, научный, литературный и т.д.), отдель но или в коллекшиях;

(1) почтовые марки, налоговые и аналогичные марки, отдельно или в коллекциях; 
(k) articles of furniture more than one hundred years old and old musical instruments.

\section{Article 2}

1. The States Parties to this Convention recognize that the illicit import, export and transfer of causes of the cultural property is one of the main tage of the countries of the cultural herithat international co origin of such property and the most efficient co-operation constitutes one of try's cultural prope sulting therefrom.

2. To this end, the States Parties undertake to oppose such practices with the means at their to posal, and particularly by means at their dis putting a stop to current practioving theircauses, to make the necessary reparation, and by helping

\section{Article 3}

The import, export or transfer of ownership of culadopted under this Conventrary to the provisions thereto, shall be ilicit.

\section{Article 4}

The States Parties to this Convention recognize that for the purpose of the Convention property which the cultural herita wing categories forms part of

(a) Cultural property created by the individual of collective genius of nationals of the State conto the State cultural property of importance ritory of that State breated within the tersiateless persons by foreign nationals or tory;

b) cultural property found within the national ter-

c) cultural property acquired by archaeological the consent of natural science missions, with country of origin competent authorities of the

(d) cultural property of such property: of a freely agreed which has been the subjec:

(e) cultural property exchange;

ased legally with the consed as a gift or purchauthorities of the countrent of the competent property. especial (historico, artístico, cientffico, litesellos de correo sueltos o en colecciones; sueltos o en colecciones.

colecciones:

ficos y cinematográs fonográficos, fotográ.

k) objeto de mobraficos: anos e instriliario que tengan más de 100

Ar:iculo 2

1. Los Estados Partes en la presente Conven cion reconocen que la imporacior, la exporialos bienes culturales de propiedad iltcitas de sas principales dei constituyen una de las caunio cultural de los malsos deciento dei patrimobienes, y que una palses de origen de dichos constituye uno de los medios intemacional proteger sus bienes medios más eficaces para todos los peligros que entrales respectivos contra que entranan aquellos actos.

prometen a combeto, los Estados Partes se comdios de que dispongan prácticas cor los mesus causas, deteniendo sobre toco suprimiendo efectuar las reparaciones curso y ayudando a

\section{Articulo 3}

Son illeitas la importacion, la exportacion y la transferencia de propiedad de los bienes cultu. rales que se efectden infringiendo las dispositud de la presente

\section{Articulo 4}

Los Estados Partes en la presente Convencion foconocen que para los efectos de la misma, Estado los del patrimonio cultural de cada rias enumeradas a continuacion: a las catego-

bienes culturaies debidos al genio individua: se trate para ese mismo culturales imporantes creados otros palses territorio por nacionales de el; $\quad$ palses o por apatridas que residan en nacional.

bienes culturales adquiridos por misiones ar queologicas, etnologicas o de ciencias natura les con el consentimiento de las autoridades competentes del pafs de des autoridades

d) bienes culturaies de origen de esos bienes intercambios libre hayan sido objeto de

e) bienes culturasente consentidos; - adquiridos legaluidos a título gratuito miento de lagalmente con el consentipals de orizen de 
( $j$ j archives, $y$ compris les archives phonographiques, photograpriques et cinématographiques :

(k) objets d'ameublement ayant pius de cent ans d'age et instruments de musique anciens. (j) архнвы, вхлючая фоно-, Фото- и кнноархнвы; (k' мебель более чем 100-летней давности и старинные музыхальные инструменты.

\section{Article 2}

1. Les Etats parties à la présente Convention reconraissent que ''importation, l'exportation e: le :ransiert de propriété illicites ces biens cu:iureis constituent !'une des causesprincipaies de l'appauvrissemen: du patrimoine culture! des pays d'origine de ces biens, et qu'une collaboration intemationale constitue i'un des moyens ies plus efficaces de protéger leurs biens cultureis respectifs contre tous les dangers qui en sont les conséquences.

2. A cette fin, les Etats parties s'engagent à combattre ces pratiques par les moyens dont ils d:sposent, notamment en supprimant leurs causes, en arretant leur cours et en aidant à effectuer les réparations qui s'imposent.

\section{Article 3}

Sout illicites l'importation, l'exportation et le trans fert de propriété des biens culturels, effectués contrairement aux dispositions prises par les Etats parties en vertu de la présente Convention.

\section{Anticle 4}

Les Etats parties à la présente Convention resonnaissent qu'aux fins de ladite convention, les biens culturels appartenant aux categories ci-apres font partie du patrimoine culture! de chaque Etat :

(a) biens culturels nés du génie individuel ou collec:: de ressortissants de l'Etat considéré et biens culturels importants pour l'Etat considéé, créés sur le territoire de cet Etat par des ressortissants étrangers ou par des apatrides résidant sur ce territoire;

(b) biens culturels trouvés sur le territoire national ;

(c) biens culturels acquis par des missions archeologiques, ethnologiques ou de sciences naturelles, avec le consentement des autorités compétentes du pays d'origine de ces biens :

(d) biens culturels ayant fait l'objet d'échanges librement consentis :

(e) biens culturels reçus à titre gratuit ou achetés légalement avec le consentement des autorités compétentes du pays d'origine de ces biens.

\section{Cтатья 2}

1. Государства - участники настоящей Конвенции признарт, что незаконные ввоз, вывоз и передача права собственности на кулітурные ценности являются одной из главных прнчнн обеднения культурного наследия стран пронсхохдения этих ценностей и что международное сотрудничество является одним из нанболее действенных средств обеспечения охраны принадлехаших нм культурных 山енностей от всех связанных с этим опасностей.

2. С этой целью государства-участники обязуются противодействовать, имеџщнмися в их распоряжения средствами, полобной прахтике, искореняя ее причины, прекращая ее осуществление и помогая произвдить необходимое возмещение.

\section{Cтатья.3}

Счнтахтся незаконными ввоз, вывоз и передача права собственности на культурные ценности, совершенные в нарушение правил, принятых государствами-участниками в соответствин с настоящей Конвенцией.

\section{C-arsr 4}

Государства - участники настоящей Коньенции признают, что в соответстзии с челями настояшей Конвеншии культурное наслелие каждого государства включает перечисленные ниже категорин ченностей:

(a) культурные ченности, созданные отдельными лицами или коллективами лиц, являхшихся гражданами данногс государства, и культурные ченности, имеюшие важное значение дтя данного государства и созданные на территорин этого государства иностранными гражданами или личами без гражданстза, проживаюшими на территории данного государства;

(b) культурные ценности, об̆наруженные на национальной террнтории;

(c) культурные ценности, приобретенные археологическими, этнологическими и естественнонаучными экспедициями с согласия компетентных властей страны, откуда пронсходят эти иенности;

(d) культурные ченности, приобретенные в результате добровольных обменов;

(е) хультурные ченности, полученные в качестве дара или законно купленные с согласия компетентных властей страны, откуда происходят эти иенности. 


\section{Article 5}

To ensure the protection of their cultural property against illicit import, export and transfer of ownership, the States Parties to this Convention under:ake, as appropriate for each country, to set up within their territories one or more national services, where such services do not already exist, for the protection of the cultural heritage, with a qualified staff sufficient in number for the effective carrying out of the following functions:

(a) Contributing to the formation of draft laws and regulations designed to secure the protection of the cultural heritage and particularly prevention of the illicit import, export and transfer of ownership of important cultural property;

(b) establishing and keeping up to date, on the basis of a national inventory of protected property. a list of important public and private cultural property whose export would constitute an appreciable impoverishment of the national cultural heritage;

(c) promoting the development or the establishment of scientific and technical institutions (museums, libraries, archives, laboratories, workshops...) required to ensure the preservation and presentation of cultural property;

(d) organizing the supervision of archaeological excavations, ensuring the preservation "in situ" of certain cultural property, and protecting certain areas reserved for future archaeological research:

(e) establishing, for the benefit of those concerned (curators, collectors, antique dealers, etc.) riles in conformity with the ethical principles set forth in this Convention; and taking steps to ensure the observance of those rules:

(f) taking educational measures to stimulate and develop respect for the cultural heritage of all States, and spreading knowledge of the provistions of this Convention:

(g) seeing that appropriate publicity is given to the disappearance of any items of cultural property.

\section{Articulo 5}

Para asegurar la proteccion de sus bienes culiu rales contra la importación, la exporacion la transferencia de propiedad ilfcitas, los Es:ado Partes en la presente Convencion se obilizan a establecer en su tertitorio, en las condiciones apropiadas a cada pals, uno o var:os servic:os de proteccion del patrimonio cuitural si esos servicios no existen aln, dotados de personai competente y en numero suficiente para garantizar de manera eficaz las funciones que se indican a continuacion:

a) contribuir a la preparación de los provec:os de textos legislativos y reglamentarios que permitan la protección del pa:rimonio cultu ral y de un modo especial la represion de las importaciones, exportaciones y transie. rencias de propiedad illcitas de los bienes culturales importantes:

b) establecer y mantener al dfa, a partir de un inventario nacional de proteccion, la lista de los bienes culturales imporiantes pablicos y privacios, cuya exportación cons:: tuirla un empobrecimiento considerabie de: patrimonio cultural nacional.

c) fomentar el desarrollo o la creación de las instituciones cientfficas y técnicas (museos bibliotecas, archivos, laboratorios, talleres, etc.). necesarias para garantizar la conser. vacion y la valorizacion de los bienes cultu. rales;

d) organizar el control de las excavaciones ar, queologicas, garantizar la conservacion "in situ" de determinados bienes culturales y proteger ciertas zonas reservadas para futuras investigaciones arqueologicas;

e) dictar, con destino a las personas interesa das (direc:ores de museos, coleccionistas, anticuarios, etc.) normas que se ajusten a los principios éticos formulados en la pre. sente Convencion y velar por el respeto de esas normas:

f) ejercer una accion educativa para estimula: y desarrollar el respeto al patrimonio culturai de todos los Estados y difundir ampiiamente las disposiciones de la presente Convencion;

g) velar por que se de la publicidad apropiada a todo caso de desaparicion de un bien cultural.

Articulo 6

Los Estados Partes en la presente Convencion se obligan:

a) A establecer un certificado adecuado, en e cual el Estado exportador autorice la exporacion del bien o de los bienes culturales de que se trate $y$ que deberá acompanar 
Afin d'assurer la protection de leurs biens culturels contre l'importation, l'exportation et le transfert de propriété illicites, les Etats parties a la présente Convention s'engagent dans les conditions appropriées à chaque pays à instituer sur leur territoire, dans la mesure oú ils n'existent pas déjà, un ou plusieurs services de protection du patrimoine culturel dotés d'un personnel qualifié et en nombre suffisant pour assurer de maniere efficace les fonc tions énumérées ci-dessous :

(a) contribuer à l'élaboration des projets de textes lég:slatifs et réglementaires en vue de permettre la protection du patrimoine culturel, et notamment la répression des importations, exportations et transferts de propriéte illicites des biens culturels importants :

(b) établir et tenir à jour, sur la base d'un inventaire national de protection, la liste des biens culturels importants, publics et privés, dont l'exportation constitue rait un appauvrissement sensible du patrimoine culturel national ;

(c) promouvoir le développement ou la création des insti:utions scientifiques et techniques (musées, bibliotheques, archives, laboratoires, ateliers. etc. ) nécessaires pour assurer la conservation et la mise en valeur des biens culturels.

(d) organiser le contrôle des fouilles archéologiques, assurer la conservation. "in situ" de cerains biens culturels et protéger certaines zones réservées à des recherches archéolog.ques futures ;

(e) établ:r. à l'intention des personnes intéressées (conservateurs, collectionneurs, antiquaires, etc.). des règles conformes aux principes éthiques formulés dans la présente Convention et ve:ller au respect de ces règles :

(f) exercer une action éducative afin d'éveiller et de développer le respect du patrimoine culture! de tous les Etats et diffuser largement la cornassance des dispositions de la présente Convention ;

(g) veiller à ce qu'une publicité appropriée soit donne à tout cas de disparition d'un bien culturel.
В челях обеспечения охраны свонх культурных 山енностей от незахонных ввоза, вывоза и передачи права собственности государства - участники настоящей Конвенции обязуются создать на своей территории, с учетом условий каждой страны, одну или несхолько национальных служб охраны культурного наследия, если такие службы еше не созданы, обеспеченные квалифицированным персоналом в колнчестве, необходимом для того, чтобы осуществлять эффективным образом перечнсленные ниже функцин:

(a) содействовать разработхе проектов захонода тельных и регламентируюших текстов, обеслечивахщих защиту культурного наследня и, в частности, пресеченне незаконных ввоза, вывоза и передачи права собственности на важные культурные ценности;

(b) составлять и обновлять на базе национального охранного реестра перечень важных культурных ченностей, госу дарственных и частных вывоз которых означал бы значительное обеднение национального культурного наследия;

(c) содействовать развитию или созданио научных н технических учрехдений (музен, бнблиотеки, архны, лабораторни, мастерсхне и т.д.), необходимых для сохранения и популяризацин культурных ценностей;

(d) организовывать хонтроль за археологичесхнми раскопхами, обеспечивать сохранение "in situ" (на сзоем месте) определенных культурных ценностей и охранять нехоторые районы, оставляемые для будущих археологических pacxonoк;

(е) устанавливать для заннтересованных лиц (хранителей, коллехнионеров, антикваров и т.л.) правила, отвечахщие этичесхим принципам, сформулированным в настоящей Хонвенции, и следить за соблюдением этих правил;

(f) осуществлять воспитательнуо деятельность с чель по пробуждения н укреплення уважения х культурному достоянию всех государств н популяризачии положений настоящей Конвенцин;

(g) следить за тем, чтобы любому случах исчезновения культурной ценности придавалась соответствудшая огласха.

\section{Статья 6}

Государства - участники настоящей Конвенции обязуются:

(a) учредить соответствуюшее свидетельство, которым государство-экспортер удостоверя ет, что оно дало разрещение на вывоз одной или нескольких культурных ценностей. Это 
(b) to prohibit the exportation of cultural property from their territory unless accompanied by the above-mentioned export certificate;

(c) to publicize this prohibition by appropriate means, particularly among persons likely to export or import cultural property

\section{Article 7}

The States Parties to this Convention undertake:

(a) To take the necessary measures, consistent with national legislation, to prevent museums and similar institutions within their territories from acquiring cultural property originating in another State Party which has been illegally exported after entry into force of this Convention, in the States concerned. Whenever possible, to inform a State of origin Party to this Convention of an offer of such cultural property illegally removed from that State after the entry into force of this Conventior in both States;

(b) (i) to probibit the import of cultural prope -5 stolen from a museum or a religious o: secular public monument or similar ins:tutior in another State Party to this Corvention after the entry into force of this Convention for the States concerned, provided that such property is documented 13 appertaining to the inventory of that ins:tution;

(ii) at the request of the State Party of orig:=, to take appropriate steps to recover anc reture any such cultural property impc:- ed atter the entry into force of this Curvention in both States concerned, proviced however, that the requesting State shai: pay just compensation to an innocent purchaser or to a person who has valid titie to that property. Requests for reccver: and return shall be made through diplomatic offices. The requesting Party shill furnish, at its expense, the documentation and other evidence necessary to establizh: its claim for recovery and return. The Parties shall impose no customs duties or other charges upon cultural propert returned pursuant to this Article. All erpenses incident to the return and delivery of the cultural property shall be borne by the requesting Party. a todos los bienes culturales regularmente exportados.

b) A prohibir la salida de su territorio de los bienes culturales no acompanados del certificado de exportacion antes mencionado.

c) A dar la oportuna difusion a esta prohibicion, especialmente entre las personas que pudieran exportar e importar bienes culturales.

\section{Artículo 7}

Los Estados Partes en la presente Convenc:on se obligan:

a) A tomar todas las medidas necesarias, conformes a la legislacion nacional, para impedir la adquisicior de bienes culturales procedentes de otro Estado Parte en la Convencion, por los museos y otras instituciones similares situados en su territorio, si esos bienes se hubieren exportado ilfcitamente después de la entrada en vigor de la Convencion; $y$ en lo posible, a informar al Estado de origen, Parte en la Convencior. de toda oferta de bienes culturales exportados ilfcitamente de ese Estado después de la entrada en vigor de la presente Convencion en ambos Estados;

b) i) A prohibir la importacion de bienes culturales robados en un museo, un monumento póblico civil o religioso, o una institucion similar, situados en el territorio de otro Estado Parte en la Convencion, después de la entrada en vigor de la misma en los Estados en cuestion, siempre que se pruebe que tales bienes figuran en el invertario de la institucion interesada;

ii) A tomar imedidas apropiadas para decomisar y restituir, a peticion del Estado de origen Parte en la Convencion, todo bien cultural robado e importado despues de la entrada en vigor de la presente Convención en los dos Estados interesados, a condicion de que el Estado requirente abone una indemnizacion equitativa a la persona que lo adquirio de buena fe o que sea poseedora legal de esos bienes. Las peticiones de comiso y restitucion deberán dirigirse al Estado requerido por via diplomática. El Estado requirente debera facilitar, a su costa, todos los medios de prueba necesarios para justificar su peticion de decomiso y restitucion. Los Estados Partes se abs. tendran de imponer derechos de aduana, u otros gravamenes, sobre los bienes 
(b) à interdire la sortie de leúr territoire des biens culturels non accompagnés du certificat d'exportation visé ci-dessus :

(c) à porter de façon appropriée cette interdiction à la connaissance du public, et en particulie: des personnes qui pourraient exporter ou importer des biens culturels.

\section{Article 7}

Les Etats parties à la présente Convention s'engagent:

(a) à prendre toutes les mesires nécessaires, conformes à la législation nationale, pour empecher l'acquisition, par les musées et autres institutions similaires situés sur leur territoire, de biens culturels en provenance d'un autre Etat partie à la Convention, biens qui auraient été exportés illicitement apress l'entrée en vigueur de la Convention: dans la mesure du possible, à informe- l'Etat d'origine, partie à la présente Convention, des offres de tels biens culturels sortis illicitement du territoire de cet Etat après l'entrée en vigueur de la présente Convention, à l'égard des deux Etats en cause

(b) (i) a interdire l'importation des biens culturels volés dans un musée ou un monument public civil ou religieux, ou une institution similaire, situés sur le territoire d'un autre Etat partie a la présente Convention après l'entrée en vigueur de celle-ci a l'égard des Etats en question, à condition çu'il soit prouvé que ce ou ces biens font partie de l'inventaire de cette institution;

(ii) a prendre des mesures appropriées pour saisir et restituer à la requête de l'Etat d'origine partie a la Convention tout bien culturel ainsi volé et importé apres l'entrée en viguear de la présente Convention à l'égard des deux Etats concernés, à condition que l'Etat requerant verse une indemnité équitable à la personne qui est acquéreur de bonne foi ou qui détient légalement la propriété dece bien. Les requetes de saisie et de restitution doivent etre adressées à l'Etat requis par la voie dipiomatique. L'Etat requérant est tenu de fournir, a ses frais, tout moyen depreuve nécessaire pour justifier sa requete de saisie et de restitution. Les Etats parties s'abs tiennent de frapper de droits de douane ou d'autres charges les biens culturels restitués en conformité avec le présent article. Toutes les dépenses afférentes à la restitution du ou des biens culturels en question sont à la charge de l'Etat requérant. свидетельство должно прилагаться $\mathrm{x}$ одной или несхольким культурным ценностям, вывозимым в соответствни с сушествуотими правилами;

(b) запретить вызоз со своей территорни культурных иенностей, К которым не прилохено вышеупомянутое свидетельство;

(c) соответствуошим образом довести настоятее запрещение до сведения обществснности и, в частности, лиц, которые могут вывозить или ввозить культурные ценности.

Crars\& 7

Государства - участники настоящей Конденцин обязухтся:

(a) прннимать все необходнмые меры, в соответствни с национальнын законодательством, направленные на предотврашение прнобретення музеями и другими аналогичныхи учрехдениями, расположенными на их террнториях, культурных иенностей, пронсходящих нз дру гого государства - участниха Конвенияи, хоторые были незахонно вывезены после вступления в силу настоящей Конвенцни. Всяхнй раз, когда это возмохно, ннформнровать государство, отхуда пронсходнт эта культурная ценность н которое является уцастником настоящей Конзенцин, о предложенни вернуть годобнуд культурнур ценность, незаконно вывезеннух из этого государства после вступления в силу настоящей Конвенцни в обонх госу дарствах;

(b) (i) запрещать ввоз культурных ценностей, похнщенных из музея или религиозного, нли сзетсхого исторнчесхого памятника, или подобного учреждения другого государства - участниха настоящей Конвенцин после вступления настоящей Конвемцин в силу. в заннтересованных гисударствах, дри условин, что такая ценность числится в описи предметов, прнкадлежащих данному учреждених;

(ii) по требованих государства-участинка предпрниматs соответствудшне шаги для обнаружения и возвращеняя лхбой подоб̆ой хультурной ценности, ввезенной после вступления настоящей Конвенцин в силу в обоих заннтересованных государствах, прн условин, однахо, что государство, обращахщееся с просьбой, выплачнвает справедтнву компемсацию добросовестному покупатель или лицу, которое имеет действительное правс иа эту ченность. Просьбы относительно розысха и возвратения направляхтся через дипломатические каналы. Требухшал сторона представляет за свой счет .лихументацих и другне доказательства, иеобходимые для устано:леняя права 1 требование в отнотенин розысха н 


\section{Article 8}

The States Parties to this Convention undertake to impose penalties or administrative sanctions on any person responsible for infringing the prohibitions referred to under Articles $6(b)$ and $7(b)$ above.

\section{Article 9}

Any State Party to this Convention whose cultural patrimony is in jeopardy from pillage of archaeological or ethnological materials may call upon other States Parties who are affected. The States Parties to this Convention undertake, in these circumstances, to participate in a concerted international effort to determine and to carry out the necessary concrete measures, including the control of exports and imports and international commerce in the specific materials concerned. Pending agreemunt each State concerned shall take provisional measures to the extent feasible to prevent irremediable injury to the cultural heritage of the requesting State.

\section{Article 10}

The States Parties to this Convention undertake:

(a) To restrict by education, inf ormation and vigilance, movement of cultural property illegally removed from any State Party to this Convention and, as appropriate for each country, oblige antique dealers, subject to penal or administrative sanctions, to maintain a register recording the origin of each item of cultural property, names and addresses of the supplier, description and price of each item sold and to inform the purchaser of the cultural property of the export prohibition to which such property may be subject;

(b) to endeavour by educational means to create and deveiop in the public mind a realization of the value of cultural property and the threat to the cultural heritage created by theft, clandestine excarations and illicit exports. culturales restituidos con arreglo al presente artículo. Todos los gastos correspondientes a la restitución del o de los bienes culturales en cuestion, correrá a cargo del Estado requirente.

\section{Articulo 8}

Los Estados Partes en la presente Convenc:on se obligar a imponer sanciones penaies o acminis. trativas a toda peruona responsable de habe: infringido las pronibiciones contenidas en ei aparta. co b) del articulo 6 y el aparado b) dei articuic :

\section{Artículo 9}

Todo Estado Parte en la presente Convencion, cuyo patrimonio cultural se encuentra en peígro, a consecuencia de pillajes arqueologicos o etnclogicos podrá dirigir un llamamiento a los Estados interesados. Los Estados Partes en la presente Convencion sc comprometen a participar en cualquier operacion interacional conceracia en esas circunstancias, para determinar y apicar las medidas concretas necesarias, inciuso el control de la exportación, la imporacion y el comercio internacional de los bienes cuituraies de que concretamente se :rate. Mientras se :ransmita el establecimiento de un acuercio, cacia Estado interesado tomará disposiciones provis:onales, en cuanto sea posible, para evitar que el patrimonio cultural del Estado peticionar:o sut:a canos irreparables.

\section{Articulo 10}

Los Estados Partes en la presente Convenc:on se obligan:

a) A restringir, por medio de la educacior, de la informacion y de la vigilancia, ia transfeŕencia de bienes culturales ilegaimente sacados de cualquier Estado Parte er la presente Convencion y a obligar a los ant:cuarios, en la forma perinente de cacia pals y bajo pena de sanciones penaies 0 acministrativas, a llevar un registro que mencione la procedencia de cada bien cultura;, el nombre y la dirección del proveedor, la descripcion y el precio de cada bien vendido, $y$ a informar al comprador del bien cultural de la prohibicion de exportacion de çue puede ser objeto ese bien.

b) A esforzarse, por medio de la educacior, en crear y desarrollar en el poblico el sent:miento del valor de los bienes culturaies $y$ del peligro que el robo, las excavaciones clandestinas y las exportaciones ilfcitas representan para el patrimonio culturai. 
возврашення. Стороны не облагахт нихаКнми таможеннымн сборамн или другими сборами культурные ценности, возвращаемые в состветствни с настоящей статьей. Ecе расходы, связанные с возврапением одной нли нескольких упомянутых культурных ценностей, несет требухшая сторона.

\section{Article 8}

Les Etats parties à la présente Convention s'engagen: à $i$-apper de sanctions pénales ou acministratives :oute personne responsable d'une infraction aux inerdictions prévues aux articles 6 (b) e: ? (b) c:dessus.

\section{Article 9}

Tout Eta: partie à la présente Convention et cont le patr:moine culturel est mis en danger par certains pillages archéologiques ou ethnologiques peut faire appel aux Etats qui sont concernés. Les Etats parties à la présente Convention s'engagent à participer a toute opération internationale concerté dans ces circonstances, en vue de déterminer et d'appliquer les mesures concrètes nécessaires, y compris le controle de l'exportation, de l'importation et du commerce international des biens culturels spécifiques concernés. Er attendant un accord, chaque Etat concerné prendra, dans la mesure du possible, des dispositions provisoires pour prévenir un dommage :rémédiabie au patrimoine culturel de l'Etat demandeur.

\section{Article 10}

Les Eta:s parties à la présente Convention s'engagen:

(a) à restreindre par l'éducation, l'information et la v:gilance, les transferts de biens culturels ilégalement enlevés de tout Etat partie à la presente Convention et, dans les conditions appropriées à chaque pays, à obliger, sous peine de sanctions pénales ou administratives, les ant:quaires à tenir un regisire mentionnant la provenance de chaque bien culturel, le nom. et l'adresse du fournisseur, la description e: le pr:x de chaque bien vendu, ains: qu'à informe: l'acheteur du bien culturel del'interdiction d'exportation dont ce bien peut être l'objet :

(b) à s'efforcer, parl'éducation, decréer et de développer dans le public le sentiment de la valeurdes biens culturels et du danger que le vol, les fourlles clandestines et les exportations illicites représentent pour le patrimoine culturel.

\section{Статья \&}

Государства - участники настоя пей Конвенции собзуются подвертать уголовному нля администраГивному нахазания всех лиц, ответственных за нарушенне запрещений, предусмотренныхвыще в статьях 6 (b) и 7 (b).

\section{Cratbs 9}

.Iюболе государство - участник настояшей Конвенчин, культурное наследие которого подвергается опасности хшения археологических или этнологических материалов, может обратнться к другим государствам-участникам, которых это касается. Государсгва - участники настоящей Конвенцин обязуются в таких случаях участвовать в согласованном международном усилин по определению н осушествленни необходимых конкретных мер, вк.точая хонтроль за вывозом, ввозом и нехдународной торговлей соответствующими хонхретными культурными ценностями. До соглашения каждое заннтересованное государство прелпринимает предварнтельные меры, в возможных пределах, направленные на предупреждение нанесения непоправимого ущерба культурному наследию государства, обращахиегося с просьбой.

\section{Cтатья 10}

Государства - участники настоящей Конвенцин оо̆язу хоск:

(a) ограничивать посредством образования, инФормачин и бдительности перевозку хультурных ценностей, незаконно вывезенных из лобого государства - участника настоящей Конвенции, и в завнсимости от условий хаждой страны вменить антикварам в обязанность, под угрозой утоловных санкций, ведение реестра, в котором указывать пронсхождение каждой культурной ценности, фамилию и адрес поставшика, описание и стоимость хахдой проданной вешн, а также информнровать похупателей культурных ценностей о возможном распространения запрешення о вывозе на эти ценности;

(b) стремиться с помошь по посветительныхсредств создавать н развивать в тлазах общественного мнения сознание значения хультурных ценностей и угрозы культурному наслелир, которух представляют кража, тайные расхопки и незахонный вывоз. 
возвратения. Стороны не облагахт нихакими таможенными сборами или другими сборами культурные ценности, воэврашаемые в состветствнн с настоящей статьей. Ece расходы, связанные с возвраtпением одной или нескольких упомянутых культурных ценностей, несет требуршая сторона.

Article 8

Les Etats parties à la présente Convention s'engagen: à irapper de sanctions pénales ou acministrat:ves oute personne responsable d'une infraction aux $n$. erdictions prévues aux articles 6 (b) e: 7 (b) c:dessus.

\section{Article 9}

Tout Eta: partie à la présente Convention et cont le patrimone culturel est mis en danger par certains pillages archélogiques ou ethnologiques peut faire appel aux Etats qui sont concernés. Les Etats parties à la présente Convention s'engagent à participer à toute opération internationale concertée dans ces circonstances, en vue de déterminer et c'appliquer les mesures concrètes nécessaires, y compris le controle de l'exportation, de l'importation et du commerce international des biens culturels spécifiques concernés. Es attendant un accord, chaque Etat concerné prendra, dans la mesure du possible, des dispositions provisoires pour prévenir un dommage :rrémédiable au patrimoine culturel de l'E:at demandeur.

\section{Article 10}

Les Eta:s parties à la présente Convention s'engagent:

(a) d restreindre par l'éducation, l'information et la viglance, les transferts de biens culturels :11également enlevés de tout Etat partie à la presente Convention et, dans les conditions appropriées à chaque pays, à obliger, sous pe:ne de sanctions pénales ou administratives, les ant:quaires à tenir un regisire mentionnant la provenance de chaque bien culturel, le nom. et l'adresse du fournisseur, la description e: le pr:x de chaque bien vendu, ainsi qu'à informer l'acheteur du bien culturel del'interdiction d'exportation dont ce bien peut être l'obje:

(b) à s'efforcer, parl'éducation, decréer et de développer dans le public le sentiment de la valeurdes biens culturels et du danger que le vol, les fourlles clandestines et les exportations illicites représentent pour le patrimoine culturel.

\section{Cтатья 8}

Государства - участники настоящей Конвенции собязуются подвертать уголовному нля администраГивному наказанию всех лин, ответственных за нарушение запрешений, предусмотренныхвыше статьях 6 (b) и 7 (b).

\section{Cтатья 9}

Inōoe государство - участник настояшей Конвеншин, культурное наследие которого подвергается опасности хншеня археологических или этнологи ческих материалов, может обратиться к другим государствам-участникам, которых это касается. Государсзва - участники настоящей Конвенцин об̆зуотся в таких случаях участвовать о согласованном международном усилин по определению н осуществленню необходимых конкретных мер, включая контроль за вывозом, ввозом и мехдународной торговлей соответствуюшими хонхретными культурными ценностями. До соглашения хаждое заннтересованное государство предпринияает предварительные меры,в возможных пределах, направленные на предупреждение нанесения непоправимого ущерба культурному наследно государства, обращахиегося с просьбой.

\section{Статья 10}

Государства - участники настоящей Конвенции оо̆язуютск:

(a) ограничнвать посредством образования, инФормацин и бдительности перевозку культурных ценностей, незаконно вывезенных из лобого государства - участника настоящей Кон венцин, и в зависимости от условий хахдой страны вменнть антикварам в обязанность, под угрозой уголовных санкинй, ведение реестра, в котором указывать пронсхожденне каждой культурной ченности, фамилню и адрес поставшика, описание и стонмость хахдой проданной вещи, а также информнровать покупателей культурных ценностей о возмох ном распространения запрещення о вывозе на эти ценности;

(b) стремиться спомошь поросветительныхсредств создавать и развивать в глазах общественного мнения сознание значения хультурных ценностей и угрозы культурному наслелир, которую представляот кража, тайные расхопхи и незахонный вывоз. 
Sont considérés comme illicites l'exportation et le transfert de propriété forcés de biens culiurels résultant directement ou indirectement de l'occupa tion d'un pays par une puissance étrangère.

\section{Article 12}

Les Etats parties à la présente Convention respecteront le patrimoine culturel dans les territoires dont ils assurent les relations internationales et prendront les mesures appropriées pour interdire et empecher l'importation, l'exportation et le transfert de propriété illicites des biens culturels dans ces territoires.

\section{Article 13}

Les Etats parties à la présente Convention s'engagent par ailleurs dans le cadre de la législation de chaque Etat :

(a) à empecher, par tous moyens appropriés, les transferts de propriété de biens culturels tendant a favoriser l'importation ou l'exportation illicites de ces biens ;

(b) à faire en sorte que leurs services compétents collaborent en vue de faciliter la restitution, a qui de droit, dans les délais les plus rapides des biens culturels exportés illicitement ;

(c) a admettre une action de revendication de biens culturels perdus ou volés exercée par le propriétaire légitime ou en son nom :

(d) à reconnafre, en outre, le droit imprescriptible de chaque Etat partie à la présente Convention, de classer et déclarer inaliénables certains biens culturels qui, de ce fait, ne doivent pas etre exportés, et a faciliter la récupération par l'Etat intéressé de teis biens au cas où ils auraient été exportés.
Счнтаптся тахже незаконными прннуднтельные вывоз и передача права собственности на культурные ценности, являюшиеся прямым или косвенным результатом оккупачия страны иностранной дерхавой.

\section{Cтатья 12}

Государства - участники настоящей Конвенция будут уважать хультурное достояние на территорнях, за ведение внешних сношений которых они несут ответственность, и прнмут соответствухшне меры для запрещения и предупрехдения незахонных ввоза, вывоза и передачи права собственности на культурные ценности на их террнториях.

\section{Crats 13}

Государства - участники настоящей Конвеншни обязуются, кроме того, в соответствии с захонодательством хаждого государства:

(a) предупреждать всеми надлехащими средствами передачу права собственности на хультурные ценности, способствуршур незахоннщи ввозу или вывозу этих иенностея̆;

(b) прннимать меры х тому, чтобы их компетентные службы сотрудничали в целях по возмохности нанболее быстрого возвращения захонным собственнихам незахонно вывезеннмх кульгурных ценностей;

(c) допусхать предзявление исха, направленного на возвращение утерянных или ухраденных культурных иенностей, со сторонщ или от имени законного собственниха;

(d) признавать, кроме того, неотзеллеме право хаждого государства - участияка настоящей Конвенцин классифицировать и обзявлять некоторые культурные ценности неотчуддаемыин, хоторые ввиду этого не долтны вывозиться, и содействовать возврадеяио заннтересованным государствам тахих культурных ценностей в том случае, если они были ранее вывезены.

\section{Cratbr 14}

Іля того чтобы предотвратить незаконный вывоз и выполнить обязательства, связанные с осудествлением этой Конвенции, Каждое государство участних Конвенцин по мере свонх возмохностей должно предоставлять национальным слухбам по охране культурното наследня достаточные средства и в случае необходимости может создавать Фонды в выщеупомянутых целях. 


\section{Article 15}

Nothing in this Convention shall prevent States Parties thereto from concluding special agreements among themselves or from continuing to implement agreements already concluded regarding the restitution of cultural property removed, whatever the reason, from its territory of origin, before the entry into force of this Convention for the States concerned.

\section{Article 16}

The States Parties to this Convention shall in their periodic reports submitted to the General Conference of the United Nations Educational, Scientific and Cultural Organization on dates and in a manner to be determined by it, give information on the legislative and administrative provisions which they have adopted and other action which they have taken for the application of this Convention, together with details of the experience acquired in this field.

\section{Article 17}

1. The States Parties to this Convention may call on the technical assistance of the United Nations Educational. Scientific and Cultural Organization, particularly as regards:

(a) Information and education;

(b) consultation and expert advice;

(c) co-ordination and good offices.

2. The United Nations Educational, Scientific and Cultural Organization may, on its own initiative conduct research and publish studies on matters relevant to the illicit movement of cultural property:

3. To this end, the United Nations Educational, Scientific and Cultural Organization may also call on the co-operation of any competent non-governmental organization.

4. The United Nations Educational, Scientific and Cultural Organization may, on its own initiative, make proposals to States Parties to this Convention for its implementation.

5. At the request of at least two States Parties to this Convention which are engaged in a dispute over its implementation, Unesco may extend its good offices to reach a settlement between them.

\section{Artículo 15}

Ninguna disposicion de la presente Convencion impedirá que los Estados Partes en ella concier. ten entre sí acuerdos pariculares o sigan aplicando los ya concertados sobre la restitucion de los bienes culturales salidos de su territorio de origen, cualquiera que fuère la razon, antes de haber entrado en vigor la presente Convencion para los Estados interesados.

\section{Artículo 16}

Los Estados Partes en la presente Convencion indicarán, en los informes periodicos que presentarán a la Conferencia General de la Organizacion de las Naciones Unidas para la Educacion, la Ciencia y la Cultura, en las fechas y en la forma que ésta determine, las disposiciones legislativas y reglamentarias, asi como las demás medidas que hayan adoptado para aplicar la presente Convencion, con detalles acerca de la experiericia que hayan adquirido en este campo.

\section{Artículo 17}

1. Los Estados Partes en la presente Convención podrán recurrir a la ayuda técnica de la Organizacion de las Naciones Unidas para la Educacion, la Ciencia y la Cultura, sobre todo en lo que respecta a:

a) la informacion y la educacion;

b) la consulta y el dictamen de expertos;

c) la coordinacion y los buenos oficios.

2. La Organizacion de las Naciones Unidas para la Educacion, la Ciencia y la Cultura podrá por su propia iniciativa, realizar investigaciones y publicar estudios sobre asuntos relacionados con la circulacion illcita de bienes culturales.

3. Con este objeto, la Organizacion de las Naciones Unidas para la Educacion, la Ciencia y la Cultura podrá también recurrir a la ccoperacion de toda organizacion no gubernamental competente.

4. La Organizacion de las Naciones Unidas para para la Educacion, la Ciencia y la Cultura podra, por propia iniciativa, presentar propuestas a los Estados Partes con miras al cumplimiento de la presente Convencion.

5. A peticion de dos Estados Partes, por 10 menos, que se hallen empenados en una controversia respecto de la aplicacion de la presente Convencion, la Unesco podrá ofrecer sus buenos oficios para llegar a un arreglo entre ellos. 
Rien, dans la présente Convention, n'empeche les Etats qui y sont parties de conclure entre eux des accords particuliers ou de poursuivre la mise à exécution des accords déjà conclus concernant la restitution de biens culturels sortis de leur territoire d'origine, pour quelçue raison que ce soit, avant l'entréeen vigueur de la présente Convention ect: les Etats intéressés.

\section{Anticle 16}

Les Etats parties à la présente Convention indique$\because n t$ zans des rapports périociques qu'ils présente-ant d la Conférence générale de l'Organisation des Vaitors Unies pour l'éducation, la science et lacul:ure, aux dates et sous la forme qu'elle détermiiera, les dispositions législatives et réglementaires -t 'es autres mesures qu'ils auront adoptées pour ''application de la présente Convention, ainsi que des précisions sur l'expérience qu'ills auront acquise dans ce domaine.

\section{Article 17}

$\therefore$ Les Etats parties à la prés ente Convention peuvent fa:re appel au concours technique de 1:Organisa:ion des Nations Unies pour l'éducation, la science et la culture, notamment en ce qui concerne :

'a) l'information et l'éducation ;

(b) la consultation et l'expertise ;

' $c$ ' la coordination et les bons offices.

2. L'Organisation des Nations Unies pour l'éducation, la science et la culture peut, de sa propre int:at:ve, entreprendre des recherches et publier des études sur les problèmes relatifs à la circulation illicite des biens culturels.

3. A cet:e fin, l'Organisation des Nations Unies pour- l'écucation, la science et la culture peut également recourir à la coopération de toute organsation non gouvernementale compétente.

4. L'Organisation des Nations Unies pour l'éducat:on, la science et la culture est habilitée à faire, de sa propre initiative, des propositions aux E: 3 ts parties en vue de la mise en oeuvre de la présente Convention.

5. A la demande d'au moins deux Etats parties à la présente Convention qu'oppose un différend relatif à la mise en oeuvre de celle-ci, l'Unesco peut offrir ses bons offices afin d'arriver à un accord entre eux.
Настоящая Конвенция никоим образом не препятствует государствам-участнихам ее заключать между собой спешиальные соглашения или продолжать применять уже заключенные соглашения о возврашении культурных ценностей, вывезенных по какнм-лио̆о прнчннам с территорни, откуда они пронсходят, до вступления настояшей Конвенини в силу в заинтересованных государствах.

\section{Статья 16}

Государства - участники настоящей Конвенцин будут сообщать в пернодических докладах, которые они представляхт Генеральной конференцин Органнзации объединенных наций по вопросам ооразования, науки н культуры в установленные ељ сроки и форме, О законодательных и регламентируџинх положениях н о других мерах, принятых ими в челях выполнения настоятей Конвенция, а также сведения об опыте, нахопленном ими в этой области.

\section{Cтатья 17}

1. Государства - участники настоящей Конвеншии могут обрашаться за тедничесхим содействнем $k$ Органнзацин объединенных наций по вопросам образования, науки н культуры, в частности, в том, что касается:

(a) ннформации и просвешения,

(b) консультаций и услуг экспертов,

(c) координачия н добрых услу:

2. Организашия обзединенных наций по вопросам образования, науки н культуры мохет по своей инишнативе проводить исследования и публиковать монография по вопросам, касахшимся незахонного перемешения культурных ценностей.

3. Вэтих целях Организация обзединенных наций по вопросам образования, науки и культуры может также прнбетать к сотрудничеству любой компетентной неправительственной организацин.

4. Организация об̄ъединенных наший по вопросам образования, науки и культуры может по своей инишиативе делать государствам-участникам предложения, направленные на выполнение настояней Конвеншии.

5. По просьбе не менее двух государств - участннков Конвенцин, между которыми возникли. разногласия отногительно ее прнменения, ЮНЕСКО может предложить свон добрые устуги для достижения соглашения между ними. 
This Convention is drawn up in English. French. Russian and Spanish, the four texts being equally authoritative.

\section{Article 19}

1. This Convention shall be subject to ratification or acceptance by States members of the United Nations Educational. Scientific and Cultural Organization in accordance with their respective consttutional procedures.

2. The instruments of ratification or acceptance shall be deposited with the Director-General of the United Nations Educational, Scientific and Cultural Organization.

\section{Article 20}

1. This Convention shall be open to accession by all States not members of the United Nations Educational, Scientific and Cultural Organization which are invited to accede to it by the Executive Board of the Organization.

2. Accession shall be effected by the deposit of ar instrument of accession with the DirectorGeneral of the United Nations Educational, Scientific and Cultural Organization.

\section{Aricle 21}

This Convention shall enter into force three months after the date of the deposit of the third instrument of ratification, acceptance or accession, but only with respect to those States which have deposited their respective instruments on or before that date. It shall enter into force with respect to any other State three months after the deposit of its instrument of ratification, acceptance or accession.

\section{Article 22}

The States Parties to this Convention recognize that the Convention is applicable not only to their metropolitan territories but also to all territories for the international relations of which they are responsible; they undertake to consult, if necessary, the governments or other competent authorities of these territories on or before ratification, acceptance or accession with a view to securing the application of the Convention to those territories, and to notify the Director-General of the United Nations Educational, Scientific and Cultural Organization of the territories to which it is applied, the
La presente Convención está redactada et. es nol, frances, inglés y ruso. Los cua:ro te: hacen iguaimente fe.

\section{Articulo 19}

1. La presente Convención se someterá a i ratificacion o a la acepracion de los Estados Miembros de la Organizacion de las Nacione Unidas para la Educacion, la Ciencia y la C ra, con arreglo a sus procedimientos const: cionaies respectivos.

2. Los instumentos de ratificacion o de ac tacion se depositarán en poder del Director General de la Organizacion de las Naciones Unidas para la Educacion, la Ciencia y la Cultura.

\section{Articulo 20}

1. La presente Convención estará abiera a adhesion de todo Estado no miembro de la 0 nizacion de las Naciones Unidas para la Edue cion, la Ciencia y la Cultura, invitado a adh rirse a ella por el Consejo Ejecutivo de la Organizacion.

2. La adhesion se hará mediante el depósitc de un instrumento de adhesion en poder del Director General de la Organización de las Naciones Unidas para la Educacion, la Cienc: $y$ la Cuitura.

\section{Articulo 21}

La presente Convencion entrará en vigor tres meses después de la fecha de deposito de: te: cer instrumento de ratificacion, de aceptacio: - de adhesion, pero solio respecto a los Estac que hayan depositado sus instrumentos respec vos de ratificacion, de aceptacion o de adhes: en esa fecha o con anterioridad. Para cada uno de los demás Estados, entrará en vigor tres meses después del deposito de su respec tivo instrumento de ratificacion, de aceptacion. de adhesion.

\section{Arriculo 22}

Los Estados Partes en la presente Convencibr. reconocen que ésta es aplicable no solio a sus territorios metropolitanos sino también a los territorios de cuyas relaciones internacionales están encargados, y se comprometen a consui: en caso necesario, a los gobiernos o demás autoridades competentes de los territorios me: cionados en ei momento de ratificar, acepta: - adherirse a la Convencion, o con anterior:dad, con miras a obtener la apiicación de la Convencion en esos territorios, así como a nc tificar al Director Generai de la Organizacion: 
La presente Convention es: étajlie en angiais, en espagnol, en frança:s eter russe, les qua:re :ex:es faisant également fo:

\section{Artic!e 19}

1. La présente Convention sera soumise à la ra:ification ou d l'acceptation ces Etats membres de l'Organisation des $\mathrm{Nat}$ ons Lnies pour l'educat:on, la science et la cul:ure, coniormémen: à leurs procécures constitu:ionnelles respec:ives

2. Les instruments de ratification ou d'acceptation seront céposes aupres du Directeur general de l'Organisation des Nations Unies pour !'éducation. la science et la culture.

\section{Article 20}

1. La présente Convention es: ouverte à l'achés:on de tout Etat non membre ce l'Organisation des Nations linies pour l'éducation, la science e: la culture, invité à $y$ achérer par le Conseil exécutif de l'Organisation.

2. I'achésion sefera par le dépot d'un ins:rument d'achésion aupres du Directeur généai de l'Organ:sation des Nations Unies pour l'éducation, la science et la cuiture.

\section{Article 21}

La présente Convention entrera en vigueur trois mois après la date de dépot du tro:sième ins:-ument de ratification, d'acceptation ou d'adhesion, mais uniquement à l'égard des Etats qui auront déposé leurs instruments respectifs de ratification, d'adceptation ou d'adhésion à cette date ou antérieurement. Elle entrera en vigueur pour chaçue autre Etat trois mois après le dépot deson instrument de ratification, d'acceptation ou d'adhés:on.

\section{Article 22}

Les Etats parties à la présente Convention reconnaissent que celle-ci est applicable non seulement a leurs ter-itoires metropolitains mais aussi aux territoires dont ils assurent les relations interna: onales; ils s'engagent à consulter, si nécessaire, les gouvernements ou autres autorités compétentes desdits territoires, au moment de la ratification. de l'acceptation ou de l'adhésion, ou auparavan:, en vue d'obtenir l'application de la Convention à ces terntoires, ainsi qu'à notifier au Directeur général de l'Organisation des Nations Lnies pour l'education, la science et la culture, les ter-toires li астоящая Конвенция состаялена на английсксм, испанеком, форнузасом н русском языках, причем вся четыре текста имест равнук силу.

\section{Cтатья!9}

․ Настоя̈ая Конвенция подтежи- ратификашии или принятик государстзами - членами Организа„ии оо̆зединеиных начий по вопросам ооразования, науки н кутьтуры в порядке, предусмотренном нх конституинямн.

2. Ратиф̆каинонные грамоты нли ахгы о принятии сдахтся на хранение Генеральному директору органнзации обз диненных наший по вопросам об́разования, наукн н культуры.

\section{Cะатья 20}

i. К настояшей Конзенцин может присоелинить-

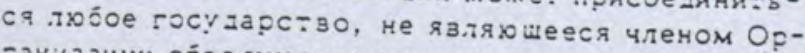
Ганизаияи об́зеднненных паций по вопросам образования, науки н культуры, которое получит от Исполнительного совета Организачин приглашенне присоелиниться $\mathrm{x}$ ней.

2. Прнсоединечие осушествляется путем сдачи ахта о присоеднненин на хранение Генеральному эирекгору Организацин обз зенненных наций по вопросам образсвания, науки н культуры.

\section{C:ars: 2:}

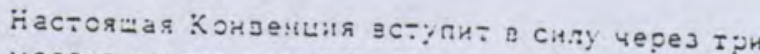
месяца со дня слачи на х:анение третьей ратификачнонной грамотst или акад о псинятии или присоединенин, но лишь в отношенни тех государсть, которые сдали на хранение свон акты о ратификаयин, принятии или прнсоедннении в указанный дечь или ранее. В отношения люёого другого госу дарста Конвенция вступает в силу через трн месяна после того, как оно сдало на хранение

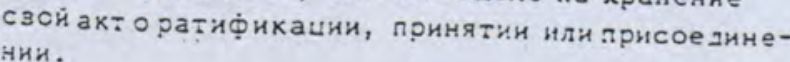

\section{C:aтbr 22}

Государстза - участиики иастояшей Конвении признают, что ее дейстан распространястся не только на территории их метрополии, но и на тероитории, за внешние сношения которых они. несут ответстзенность; Онн обязуктсн консультисоваться, если неооходимо, с правительстваМи нли С компетентными эластями указаниых территорий з момент ратиф̆ікацин, принтия или присоединения, или еще ранее, чтоб̆ опесльчить осушествленне Конвенцин на этих территорнях, а также нотифичировать Генерального директора Организацин об̆ъеднненных начий по вопрссам 
notification to take effect three months after the date of its receipt.

\section{Article 23}

1. Each State Party to this Convention may denounce the Convention on its own behalf or on behalf of any territory for whose international relations it is responsible.

2. The denunciation shall be notified by an insimment in writing, deposited with the DirectorGeneral of the United Nations Educational, Scien:ific and Cultural Organization.

3. The denunciation shall take effect twelve months after the receipt of the instrument of denunciation.

\section{Article 24}

The Director-General of the United Nations Educational, Scientific and Cultural Organization shall inform the States members of the Organization, the States not members of the Organization which are referred to in Article 20, as well as the United Nations, of the deposit of all the instruments of ratification, acceptance and accession provided for in Articles 19 and 20 , and of the notifications and jenunciations provided for in Articles 22 and 23 respectively.

\section{Article 25}

1. This Convention may be revised by the General Conference of the United Nations Educational. Scientific and Cultural Organization. Any such revision shall, however, bind only the States which shall become Parties to the revising convention.

2. If the General Conference should adopt a new convention revising this Convention in whole or in part, then, unless the new convention otherwise provides, this Convention shall cease to be open to ratification, acceptance or accession, as from the date on which the new revising convention enters into force.

\section{Article 26}

In conformity with Article 102 of the Charter of the United Nations, this Convention shall be registered with the Secretariat of the United Nations at the request of the Director-General of the United Nations Educational, Scientific and Cultural Organization. de las Naciones Lnidas para la F.ducacien, Ciencia y la Cultura, los territorios a los les se aplicará la Convencion. Esta rat:ïic: cion surtirá eiecto tres meses después de : fecha de su recepcion.

\section{Artículo 23}

1. Cada uno de los Estados Partes en la p sente Convención tendrá la facultad de cenur. ciarla en su nombre propio o en nombre de :odo territorio cuyas relaciones internaciona. tenga a su cargo.

2. La denuncia se notificará mecian:e ins:mento escrito que se depositará en pode: de Director General de la Organizacion de las Naciones Unidas para la Educacion, la Cienc y la Cuitura.

3. Lâ denuncia surt:rá efecto doce meses c pués de la recepcion de! instrimento de dent cia.

\section{Artículo 24}

El Director Ceneral de la Organizacion de la Naciones Unidas para la Educacion, la Cienc: y la Cultura informará a los Estados Miemb: de la Organizacion, a los Estados no miembr a que se refiere el artículo 20 , asf como a : Naciones Unidas, del deposito de todos los in. trumentos de ratificacion, de aceptacion o de adhesion que se mencionan en los ar:fculos : y 20, al iguai que de las modificaciones y de nuncias respectivamente previstas en los arti los 22 y 23 .

Arriculo 25

1. La Conferencia General de la Organizacio de las Naciones Unidas para la Educacion, la Ciencia y la Cultura podrá revisar la present: Convencion. Sin embargo, la revision solo o: gará a los Estados que lleguen a ser partes $\epsilon$ la Convencion revisada.

2. En caso de que la Conferencia General apruebe una nueva Convencion que constituya una revision total o parcial de la presente, y a menos que la nueva Convención disponga ot: cosa, la presente Convencion dejará de estar abierta a la ratificacion, a la aceptación 0 a : adhesion, a partir de la fecha de entrada en vigor de la nueva Convencion revisada.

\section{Arriculo 26}

Con arreglo a lo dispuesto en el artículo 102 de la Carta de las Naciones Unidas, la presen te Convención se registrará en la Secretarfa de las Naciones Unidas a peticion del Director General de la Organizacion de las Naciones Unidas para la Educacion, la Ciencia y la Cultura. 
auxquels la Convention s'appliquera, cette ratification devant prendre effet trois mois après la date de sa réception.

\section{A rticle 23}

1. Chacun des Etats parties à la présente Convention aura la faculté de dénoncer la présente Convention en son nom propre ou au nom de tout ter-itoire dont il assure les relations internationales.

2. La dénonciation sera notifiée par un instrument écrit déposé auprès du Directeur général de l'Organisation des Nations Unies pour l'éducation, la science et la culture.

3. La dénonciation prendra effet douze mois après réception de l'instrument de dénonciation.

\section{Article 24}

Le Directeur général de l'Organisation des Nations Unies pour l'éducation, la science et la culture informera les Etats membres de l'Organisation, les Etats non membres visés a l'article 20 , ainsi que l'Organisation des Nations Unies, du dépot de tous les instruments de ratification, d'acceptation ou d'adhésion mentionnés aux articles 19 et 20 , de meme que des notifications et dénonciations respectivement prévues aux articles 22 et 23 .

\section{Article 25}

1. La présente Convention pourra etre révisée par la Conférence générale de l'Organisation des Nations Unies pour l'éducation, la science et la culture. La révision ne liera cependant que les Etats qui deviendront parties à la Convention portant révision.

2. Au cas où la Conférence générale adopterait une nouvelle convention portant révision totale ou partielle de la présente Convention, et à moins que la nouvelle convention n'en dispose autrement, la présente Convention cesserait d'etre ouverte à la ratification, à l'acceptation ou à l'adhésion, à partir de la date d'entrée en vigueur de la nouvelle convention portant révision.

\section{Article 26}

Conformément à l'article 102 de la Charte des $\mathrm{Na}-$ tions Unies, la présente Convention sera enregistrée au Secrétariat des Nations Unies à la requete du Directeur général de l'Organisation ues Nations Unies pour l'éducation, la science et la culture. образования, науки и культуры о террнториях, на которых Конвенция будет осуществляться. Эта нотифихация вступает в силу через три месяна после ее получения.

\section{Cтатья 23}

1. Каждое государство - участних настояпей Конвенцин может ее денонсировать от своего имени или от нмени любой территорни, за внешние сношення которой оно несет ответственность.

2. Денонсация нотифицируется письменным ахтом, который сдается Г енеральному днректору Организацин объеднненных наций по вопросам образованяя, науки и кульгуры.

3. Денонсация вступает в силу через двенадать месяцев после получения акта О денонсацин.

\section{Статья 24}

Генеральный директор Организацин объелиненных нащий по вопросам образования, науки н культуры сообшает государствам-членам Организация, государствам, не состоящим членами Организанин, упомкнутым в статье 20, а тахже Организашия Объединенных Наций о сдаче на хранение всех актов О ратификацин, прннятин или прнсоединенин, упомянутых в статьях 19 и 20, а такхе о нотификацин и денонсациях, ухазанных в статьях 22 и 23.

\section{Статья 25}

1. Настоящая Конвенция мохет быть пересмотрена Г енеральной хонференцией Организация обзединенных наший по вопросам образования, наухи и культуры. Однако ее пересмотренный текст будет обязывать лишь те государства, хоторые станут сторонами пересмотренной Конвенции.

2. В случае, если Генеральная хонференияя примет новух конвенцию в результате палвого или частичного пересмотра настоящей Конвенцин и если новая конвенция не будет содерхать друтих указаний, насzоящая Конвеншия будет захрыта для ратификаши, прннятия или присоединения со дня вступления в силу новой хонвенияи, содерхапей пересмотренний техст.

\section{Cratba 26}

Согласно статье 102 Устава Организации Объе днненных Наций, настоящая Конвенция будет зарегистрирована в Сехретариате Организация Объеднненных Наций по просьбе Генеральното днрехтора Организация обзединенных наций по вопросам образовання, наухи н хультуры. 
Done in Paris this seventeenth day of November 1970 , in two authentic copies bearing the signature of the President of the sixteenth session of the General Conference and of the Director-General of the United Nations Educational, Scientific and Cultural Organization, which shall be deposited in the archives of the United Nations Educational, Scientific and Cultural Organization, and certified true copies of which shall be delivered to all the States referred to in Articles 19 and 20 as well as to the United Nations.

The foregoing is the authentic text of the Convention duly adopted by the General Conference of the United Nations Educational, Scientific and Cultural Organization during its sixteenth session, which was held in Paris and declared closed the fourteenth day of November 1970.

IN FAITH WHEREOF we have appended our signatures this seventeenth day of November 1970 .

The President of the General Conference

ATILIO DELL'ORO MAINI

The Director-feneral

RENE MAHEU

Certified copy

Paris,
Hecho en París en este dia diecisiete de noviembre de 1970 , en dos ejemplares auténticos que llevan la firma del Presidente de la Conferencia General, en su 16 a. reunion y de! Director General de la Organización de las Naciones Unidas para la Educacion. la Ciencia y la Cultura. ejemplares que se depositarán en los archivos de esta Organización, y cuyas copias certificadas conformes se remitirán a todos los Estados a que se refieren los art'́culos 19 y 20 , asi como a las Naciones Unidas.

Lo anterior es el texto auténtico de la Convencion aprobada en buena y debida forma por la Conferencia General de la Organizacion de las Naciones Unidas para la Educacion, la Ciencia y la Cultura, en su decimosexta reunion, celebrada en Paris y terminada el catorce de no viembre de 1970 .

EN FE DE LO CUAL estampan sus firmas, en este dia diecisiete de noviembre de 1970.

El Presidente de la Conferencia General

\section{ATILIO DELL'ORO MAINI}

El Director General

RENE MAHEU

Copia certificada conforme París, 
Fait a Paris, ce dix-septieme jour de novembre 1970 , en deux exemplaires authentiques portant la signature du Président de la Conférence générale, rénie en sa seizième session, et du Directeur général de l'Organisation des Nations linies pour l'education, la science et la culture, qui seront déposés dans les archives de l'Organisation des Nations Unies pour l'éducation, la science et la culture, et dont des copies certifieses conformes seront remises à tous les Etats visés aux articles 19 et 20 ainsi qu'dे l'Organisation des Nations linies.

Le texte qui précede est le texte authentique de la Convention dament adoptée par la Conférence généraie de l'Organisation des Nations Unies pour l'éducation, la science et la culture à sa seizième session, qui s'es: tenue à Paris et qui a été déclarée close le quatorzieme jour de novembre 1970 .

EN FOI DE QUOI ont apposé leur signature, ce dix-septième jour de novembre 1970.

Le President de la Conjërence genérale

\section{ATILIO DELL'ORO MAINI}

Le Directew geineral

RENE MAHEU

Copie certifiée conforme Paris,
Совершенов Париже, семнаднатого ноября 1970 Г.в двух аутентичных зхземплярах за подпись Председателя Генеральной хонференшни, собравшейся на шестнашату о сессио, и Генерального пиректора Организачия объедннекных наций по вопросам образования, науки и культуры; зтн экземпляры 6 дут сданы на хранение в архнв Организачин Објзеднненных наций по вопросам образования, науки н культуры н надтежатим образом заверенные копни нх будут направлены всем государствам, указанньім в статьях 19 и 20, а также Организашни Объеднненных Н ачий.

Приведенный выще техст является подлинным текстом Конвеншин, налгежащим образом прннятой Генеральной конферениней Организании объеднненных наций по вопросам образования, науки н культуры на ее шестналшатой сессии, состоявщейся в Париже и закончнвщейся четыр надиатого ноября 1970 года.

В УДОСТОВЕРЕНИЕ ЧЕГО, настоя УХ Конвенцих подписали семнадиатого ноября 1970 года.

Ipedcedaress $\Gamma$ exepassox xonфepexwu ATILIO DELL'ORO MAINI

Texepcusoxix dupexilop

RENE MAHEU
Заверенная копия

Париж

\footnotetext{
Directew de l'Office des Normes internationales et des $A$ ffaires juridiques de l'Organisation des Nations linies pow l'education, la science et la culture
} 